\title{
Vortex Properties in a Model with Local Pairs
}

\author{
Ż. Śledź, M. MierzeJeWski And M.M. MaśKA \\ Institute of Physics, University of Silesia \\ Uniwersytecka 4, 40-007 Katowice, Poland
}

\begin{abstract}
We investigate the vortex structure in the Penson-Kolb model with and without impurities. We focus on the consequences arising from the non-local field-dependent pairing interaction for the vortex profile, current distribution, and the local density of states.
\end{abstract}

PACS numbers: 74.25.Ha, 74.20.--z

\section{Introduction}

Recent developments in the experimental techniques give rise to intensive investigations of the vortex structure. In particular, scanning tunneling microscopy experiments give a new insight into the electronic structure of the vortex core $[1,2]$. The experimental results concerning the local density of states can directly be compared with the theoretical results serving as a very useful method for testing of microscopic models. Since the magnetic flux suppresses superconducting gap locally in the vortex core, one expects a presence of the bound states, at least for the isotropic $s$-wave superconductors $[3,1]$. The aim of the present work is the analysis of the vortex structure in the attractive Penson-Kolb (PK) model [4] that describes superconductivity in a system with short-range pairing interaction. In contradiction to the attractive Hubbard (AH) model, the external magnetic field explicitly enters the term responsible for superconductivity, i.e., the pair hopping interaction. Therefore, the differences between AH and PK models may show up in the electromagnetic properties $[5,6]$, especially in the vortex structure.

\section{Model and formalism}

We start with the Hamiltonian

$$
\mathcal{H}=\sum_{i, j, \sigma}\left(t_{i j} \mathrm{e}^{\mathrm{i} \Phi_{i j}}-\delta_{i j} \tilde{\mu}_{i}\right) c_{i \sigma}^{\dagger} c_{j \sigma}-\frac{1}{2} J \sum_{\langle i, j\rangle} \mathrm{e}^{2 \mathrm{i} \Phi_{i j}} c_{i \uparrow}^{\dagger} c_{i \downarrow}^{\dagger} c_{j \downarrow} c_{j \uparrow},
$$

where $t_{i j}$ is the single electron hopping integral between sites $i$ and $j, J$ is the 
nearest neighbor pair hopping interactions. $\tilde{\mu}_{i}=\mu-w_{i}$, where $\mu$ is the chemical potential and $w_{i}$ the impurity potential. In the presence of external magnetic field the single electron hopping integral acquires the Peierls factor

$$
\Phi_{i j}=\frac{e}{\hbar c} A_{i j}=\frac{e}{\hbar c} \int_{R_{j}}^{R_{i}} \boldsymbol{A} \cdot \mathrm{d} \boldsymbol{l}
$$

whereas the phase factor in the pair hopping term is twice as large. In order to investigate the vortex structure we apply a mean-field approximation

$$
\begin{aligned}
\mathcal{H}= & \sum_{i, j, \sigma}\left(t_{i j} \mathrm{e}^{\mathrm{i} \Phi_{i j}}-\delta_{i j} \tilde{\mu}_{i}\right) c_{i \sigma}^{\dagger} c_{j \sigma}-J \sum_{i}\left(c_{i \downarrow} c_{i \uparrow} \widetilde{\Delta}_{i}^{*}+c_{i \uparrow}^{\dagger} c_{i \downarrow}^{\dagger} \widetilde{\Delta}_{i}\right) \\
& -\frac{1}{2} J \sum_{\langle i, j\rangle} \mathrm{e}^{2 \mathrm{i} \Phi_{i j}}\left(n_{i j \uparrow} c_{i \downarrow}^{\dagger} c_{j \downarrow}+n_{i j \downarrow} c_{i \uparrow}^{\dagger} c_{j \uparrow}\right),
\end{aligned}
$$

where $\widetilde{\Delta}_{i}=\frac{1}{2} \sum_{\langle j\rangle_{i}} \mathrm{e}^{2 \mathrm{i} \Phi_{i j}} \Delta_{j}, \Delta_{j}=\left\langle c_{j \downarrow} c_{j \uparrow}\right\rangle, n_{i j \sigma}=\left\langle c_{i \sigma}^{\dagger} c_{j \sigma}\right\rangle$. In the following, we neglect possible magnetic correlations and assume that $n_{i j \uparrow}=n_{i j \downarrow}=n_{i j}$. We also restrict our considerations to the nearest neighbor hopping, with the hopping integral $t$ as an energy unit. The resulting Hamiltonian (2) can be diagonalized with the help of the Bogolyubov-de Gennes (BdG) equations (for the details see, e.g., Ref. [7]). Since the magnetic field enters explicitly the pair hopping term, one may expect a substantial modification of the current distribution with respect to the AH model. In order to investigate this possibility, we have calculated the current flowing from site $i$ to site $j$

$$
I_{i j}=\left\langle\frac{\partial \mathcal{H}}{\partial A_{i j}}\right\rangle=-\frac{8 \pi}{\Phi_{0}} \operatorname{Im}\left[\left(t \mathrm{e}^{\mathrm{i} \Phi_{i j}}-\frac{1}{2} J \mathrm{e}^{2 \mathrm{i} \Phi_{i j}} n_{i j}\right) n_{i j}-\frac{1}{2} J \mathrm{e}^{2 \mathrm{i} \Phi_{i j}} \Delta_{i}^{*} \Delta_{j}\right] .
$$

\section{Results and discussion}

The BdG equations have been solved for a $31 \times 31$ lattice and various values of the model parameters. For the sake of brevity we restrict the following presentation to the case $\mu=0$ and $J=t$. First, we discuss the vortex structure in the absence of impurities $\left(w_{i}=0\right)$. Let us note that replacing $\widetilde{\Delta}_{i}$ by $\Delta_{i}$ in Hamiltonian (2) would reduce the pairing term to that of the AH model. However, in the PK model the superconducting gap in the density of states is determined by $\widetilde{\Delta}_{i}$ that depends on the value of $\Delta_{i}$ on the neighboring sites and on the Peierls phase factor. We have found that qualitatively both the parameters $\Delta_{i}$ and $\widetilde{\Delta}_{i}$ exhibit the same spatial dependence. They vanish in the vortex center, and can be well fitted by the formula commonly used for the vortex profile $\left|\Delta_{i}\right|=\left|\Delta_{0}\right| \tanh r / \xi$, where $r$ is the distance from the vortex center and $\xi$ characterizes the size of the vortex core. We have found that an approximate linear relation $\left|\Delta_{i}\right| \propto\left|\widetilde{\Delta}_{i}\right|$ holds. Therefore, in the absence of impurities the vortex profiles in the PK and AH models are qualitatively the same, at least at the mean field level. However, the expression for the superconducting currents differs significantly from that obtained for the $\mathrm{AH}$ 
one. Due to the explicit field dependence of the pair hopping interaction, additional terms $\propto J$ occur in Eq. (3). These terms give a significant contribution to the superconducting current, as can be inferred from Fig. 1, where we compare the current distribution determined by Eq. (3) with a contribution that is proportional to the single electron hopping integral $I_{t}=-\frac{8 \pi}{\Phi_{0}} \operatorname{Im}\left(t \mathrm{e}^{\mathrm{i} \Phi_{i j}} n_{i j}\right)$. Therefore, for the same value of the superconducting gap, currents in the PK model are larger than the currents in the $\mathrm{AH}$ one.

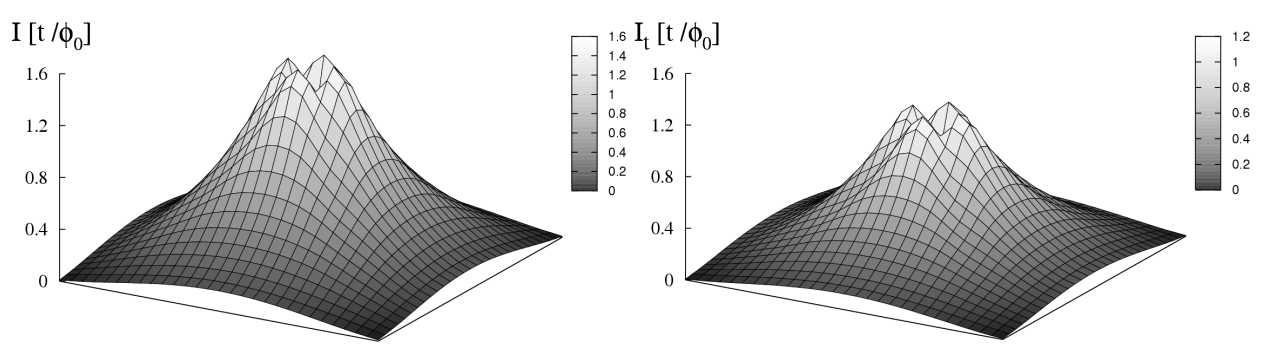

Fig. 1. The current amplitude distribution in the vicinity of the vortex core (left part). The right part shows a contribution to $I_{i j}$ that is proportional to $t$ [see Eq. (3)]. Both the currents vanish in the vortex center.

In the presence of impurities $\Delta_{i}$ and $\widetilde{\Delta}_{i}$ show different spatial dependence. It originates from the fact that $\widetilde{\Delta}_{i}$ is determined as an averaged value of $\Delta_{i}$, where the averaging is carried out over the neighboring sites. In order to investigate this problem we have considered a system containing 11 impurities with $w_{i}=2 t$. Since the vortices are strongly pinned by impurities one of them has been placed in the vortex center, whereas the remaining ones have been randomly scattered over other lattice sites. Figure 2 shows that the electron concentration is strongly modified by impurities, but the spatial dependence of $\widetilde{\Delta}_{i}$ remains almost unaffected. Additionally, we have found that impurities locally reduce the values of $\Delta_{i}$ by up to $50 \%$.

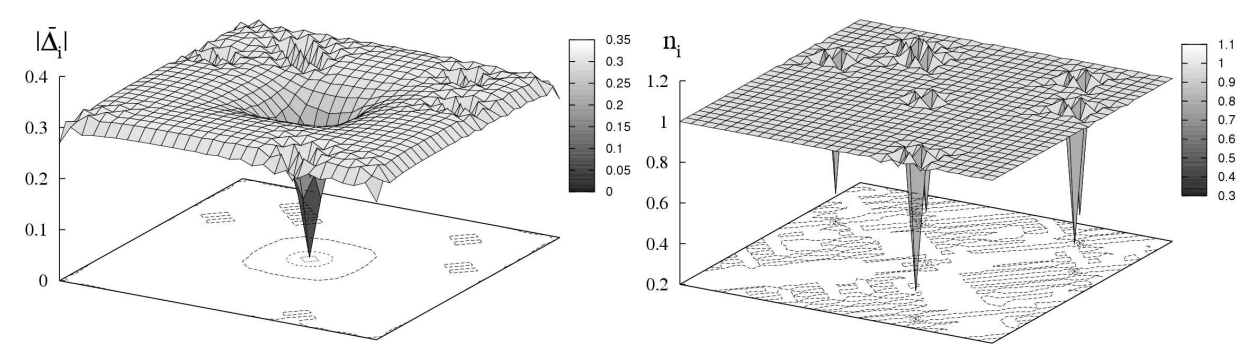

Fig. 2. Superconducting order parameter $\widetilde{\Delta}_{i}$ (left part) and the concentration of electrons $n_{i}$ (right part) in the presence of impurities. 


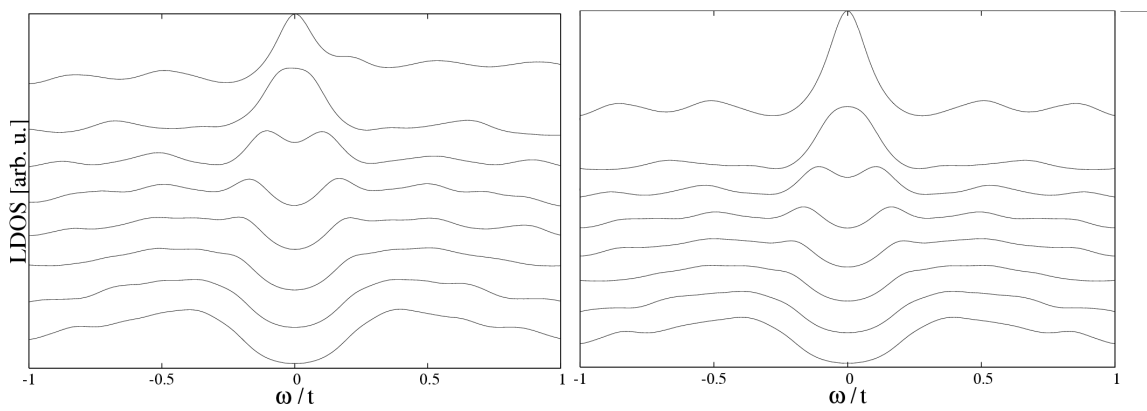

Fig. 3. Local density of states with (left part) and without the impurities (right part) for various distances from the vortex core. The topmost curves correspond to the vortex center.

Since the superconducting gap in the density of states is determined by $\widetilde{\Delta}_{i}$ and not by $\Delta_{i}$, this gap is relatively robust against the presence of impurities. This feature is reflected in the local density of states (LDOS) shown in Fig. 3. The only important consequence of the presence of impurities is a local shift of the spectral weight toward higher energies (see LDOS in the vortex center). However, independently of the presence of impurities there is a zero bias conductance peak in the vortex center, that at larger distances splits into two peaks. This splitting clearly indicates the presence of the bound states in the vortex core [3].

\section{References}

[1] H.F. Hess, R.B. Robinson, R.C. Dynes, J.M. Valles, Jr., J.V. Waszczak, Phys. Rev. Lett. 62, 214 (1989).

[2] I. Maggio-Aprile, Ch. Renner, A. Erb, E. Walker, Ø. Fischer, Phys. Rev. Lett. 75, 2754 (1995); S.H. Pan, E.W. Hudson, A.K. Gupta, K.-W. Ng, H. Eisaki, S. Uchida, J.C. Davis, Phys. Rev. Lett. 85, 1536 (2000); Ch. Renner, B. Revaz, K. Kadowaki, I. Maggio-Aprile, Ø. Fischer, Phys. Rev. Lett. 80, 3606 (1998).

[3] C. Caroli, P.G. de Gennes, J. Matricon, Phys. Lett. 9, 307 (1964).

[4] K.A. Penson, M. Kolb, Phys. Rev. B 33, 1663 (1986).

[5] W.R. Czart, S. Robaszkiewicz, Phys. Rev. B 64, 104511 (2001).

[6] M. Mierzejewski, M. Maśka, Phys. Rev. B 69, 054502 (2004).

[7] M. Maśka, M. Mierzejewski, Phys. Rev. B 68, 024513 (2003). 